\title{
Towards Developing a Practical Tool to Assist UX Evaluation in the IoT Scenario
}

\author{
Rodrigo L. A. Almeida \\ Universidade Federal do \\ Ceará \\ Fortaleza, Brasil \\ rodrigoalmeida@great.ufc.br
}

\author{
Ticianne G. R. Darin \\ Universidade Federal do \\ Ceará \\ Fortaleza, Brasil \\ ticianne@virtual.ufc.br
}

\author{
Rossana M. C. Andrade \\ Universidade Federal do \\ Ceará \\ Fortaleza, Brasil \\ rossana@ufc.br
}

\author{
Italo L. de Araújo \\ Universidade Federal do \\ Ceará \\ Fortaleza, Brasil \\ italoaraujo@great.ufc.br
}

\begin{abstract}
Internet of Things (IoT) is a paradigm that offers the users a novel and intelligent way of interacting with everyday objects. However, the complexity of an IoT scenario, involving the interaction among multiples devices, users and services, represents a challenge for the evaluation of user experience (UX). Hence, to assist in the conduction of a more effective UX evaluation in an IoT environment, this paper presents a checklist that assists evaluators with different levels of experience to perform UX evaluation in the IoT scenario. Such tool was built based on three main steps: a literature review, the UX evaluation of an IoT application, and a questionnaire answered by IoT experts.
\end{abstract}

\section{KEYWORDS}

Internet of Things, User Experience Evaluation and User Observation.

\section{INTRODUÇÃO}

A evolução tecnológica de áreas do conhecimento como redes de sensores sem fio, computação em nuvem e computação ubíqua permitiu o surgimento da Internet das Coisas, do inglês Internet of Things (IoT) [14]. IoT permite a presença pervasiva de uma variedade de coisas ao redor das pessoas. Esses objetos possuem um esquema de endereçamento único que permite a interação com outros objetos para alcançar objetivos comuns [2]. Assim, atividades do cotidiano como cozinhar, acender luzes, fazer compras podem ser auxiliadas por objetos embutidos de poder computacional e comunicação [9].

Dessa forma, em um ambiente IoT, o usuário é capaz de interagir com diversas "coisas" simultaneamente, o que resulta em uma interação complexa para o usuário. Isso ocorre porque ações e informações necessárias para as aplicações no ambiente IoT não são, muitas vezes, claras e facilmente compreendidas e utilizadas pelos usuários [11]. Por esse motivo, a literatura tem apontado que a avaliação da experiência do usuário, do inglês

In: XVII Workshop de Ferramentas e Aplicações (WFA 2018), Salvador, Brasil. Anais do XXIV Simpósio Brasileiro de Sistemas Multimídia e Web: Workshops e Pôsteres. Porto Alegre: Sociedade Brasileira de Computação, 2018. (C) 2018 SBC - Sociedade Brasileira de Computação. ISBN: 978-85-7669-435-9.
User Experience (UX), no cenário de IoT traz desafios relacionados à natureza multi-plataforma da interação, bem como à interconexão entre dispositivos, serviços e usuários [5,12]. A avaliação de UX no paradigma IoT é complexa porque precisa considerar aspectos peculiares a esses ambientes, como o número significativo de "coisas" presentes na avaliação.

Dentre esses aspectos, Roland et al. (2015) [11] destacam, por exemplo, que a interação com uma funcionalidade pode estar distribuída entre múltiplos dispositivos, o que inclui várias interfaces, usuários e regras. Além disso, o foco da UX pode ser no serviço ao invés da interação com um dispositivo, incluindo diversos tipos de contexto de uso. Além disso, tanto IoT, como UX apresentam aspectos, que no contexto da avaliação de um ambiente inteligente precisam estar estruturados para a identificação da experiência do usuário [5].

Com o intuito de auxiliar a comunidade científica a conduzir a avaliação de UX no cenário IoT, este trabalho propõe uma ferramenta de avaliação no formato de um checklist para apoiar a observação do comportamento do usuário durante a interação com uma aplicação IoT. O checklist foi construído com base na metodologia de Stufflebeam (2000) [13] com o apoio dos resultados de uma revisão da literatura, de uma avaliação de UX em um cenário IoT e de um questionário com especialistas de IoT. A ferramenta indica a possibilidade de auxiliar pesquisadores com pouca experiência na avaliação de UX no cenário de IoT.

\section{FUNDAMENTAÇÃO TEÓRICA}

A IoT é um paradigma que permite que pessoas e objetos inteligentes estejam conectados a qualquer momento, lugar, seja qual for o objeto, contanto que estejam utilizando uma rede e seus serviços [15]. A IoT tem apresentado forte influência no desenvolvimento tecnológico de vários domínios como cidades inteligentes, casas e escritórios inteligentes e na área da saúde [6]. Através desses domínios, é possível perceber a diversidade de aplicações com as quais o usuário pode interagir, resultando em tipos de experiências distintas em cada um desses sistemas.

Dessa forma, a interação em sistemas IoT apresenta desafios devido à forma como esta ocorre no cenário de IoT, bem como no contato entre o usuário e o próprio sistema. A interação é um dos desafios que surge com a IoT [1,3] e ela difere na forma como a ocorre no sistema e na forma do contato do usuário com o sistema IoT. Andrade et al. (2017) [1] e Rowland et al. (2015) [11] abordam a interação no contexto da IoT em dois níveis: 
Thing-Thing Interaction e Human-Thing Interaction. O primeiro se relaciona à intercomunicação entre os objetos inteligentes com o intuito de trocar informações e fornecer serviços aos usuários. O segundo nível se refere à interação que ocorre entre o objeto inteligente e o usuário.

A interação da thing-thing pode ser organizada em dois níveis: o de Internet e o de coisas. As questões relacionadas à Internet são centradas na conexão e relacionadas a aspectos como sincronia dos serviços, responsividade e confiabilidade da rede. Quanto ao nível das coisas, são abordados aspectos como consumo de energia, sensibilidade ao contexto, interoperabilidade e dificuldade de instalação. Todas as características dos objetos inteligentes da interação thing-thing impactam diretamente na interação do usuário com o sistema IoT [1], que consequentemente tem influência na UX, pois afetam a forma como o usuário percebe o sistema e como são atendidas suas expectativas e necessidades.

Andrade et al. (2017) [1] e Bergman e Johasson (2017) [3] listam ainda desafios na avaliação da interação na perspectiva do usuário. Segundo os autores, ambientes e sistemas IoT podem gerar diversos cenários de uso devido às possíveis combinações de objetos inteligentes, serviços e interfaces.

\section{PROCESSO DE DESENVOLVIMENTO DO CHECKLIST}

O checklist de observação do comportamento do usuário foi desenvolvido baseado nos passos da metodologia proposta por Stufflebeam (2000) [13], a qual organiza em doze fases a elaboração de um checklist. As três primeiras fases se concentram na definição do domínio, geração dos itens de verificação, a quarta e quinta fases abordam a geração das categorias e da fase seis a fase doze são realizadas a revisão, o refinamento, a formatação e a validação do checklist.

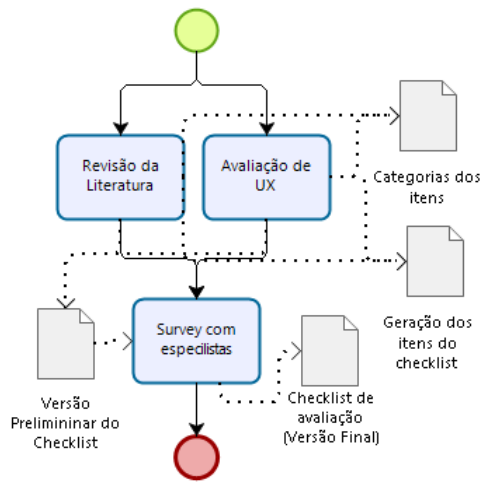

Figura 1: Passos para a construção do checklist.

Neste trabalho, aplicamos os princípios gerais da metodologia de Stufflebeam (2000) [13] que consistem na geração dos itens, classificação em categorias e a participação de especialistas de domínio na revisão e validação do instrumento. Para a geração e organização dos itens de verificação utilizamos os resultados de uma revisão da literatura e uma avaliação de UX em um ambiente IoT nas quais foi possível observar aspectos práticos relacionados com a condução da avaliação. Com a triangulação dos resultados desses métodos, aplicamos um questionário com especialistas de IoT que auxiliaram na revisão e validação do checklist. A Figura 1 descreve os métodos aplicados na geração do instrumento de avaliação e os principais resultados de cada um.

\subsection{Revisão da Literatura}

O objetivo da revisão literatura foi a identificação de estudos que realizassem a avaliação UX no contexto de IoT, para identificação de métodos de avaliação e características de IoT relevantes no contexto de UX. Para isso, foi utilizada a string descrita na Figura 2. A string foi aplicada na Scopus Digital Library resultando em um conjunto de 471 estudos. Todo o processo da revisão foi executado por um especialista em avaliações de IHC (Interação Humano-Computador), com cinco anos de experiência na área, e revisado por um especialista de IoT e outro de IHC. Após a leitura de título e resumo, seguido pela leitura completa dos trabalhos foram selecionados um conjunto de 17 estudos. Devido ao limite de espaço do artigo, os estudos identificados na revisão estão disponíveis em: https://goo.gl/KBSTQ9.

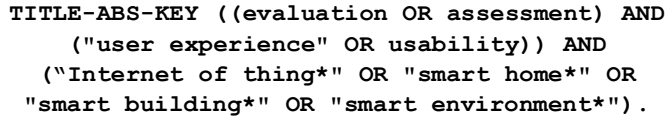

Figura 2. String de busca utilizada na revisão da literatura.

Os estudos apresentaram a predominância de utilização de métodos empíricos (94\%), como observação do comportamento do usuário e entrevista, e a avaliação de características de IoT como programabilidade (65\%) e sensibilidade ao contexto (29\%). Foram identificados alguns problemas de UX recorrentes em aplicações IoT, assim como as principais dificuldades descritas pelos avaliadores. Na Tabela 1 observamos a descrição de alguns exemplos encontrados na literatura de problemas e dificuldade no cenário descrito anteriormente.

Tabela 1: Exemplo de descrição dos problemas e dificuldades identificado na revisão da literatura

\begin{tabular}{clclc}
\hline & Problemas de UX & \multicolumn{3}{c}{ Dificuldades na execução da avaliação } \\
\hline Estudo & Descrição & Estudo & Descrição \\
\hline$[10]$ & $\begin{array}{l}\text { Dificuldades na instalação do } \\
\text { objeto inteligente no ambiente } \\
\text { IoT. }\end{array}$ & {$[4]$} & $\begin{array}{l}\text { Avaliação no ambiente } \\
\text { controlado pode destoar do } \\
\text { ambiente de uso real do } \\
\text { usuário. }\end{array}$ \\
\hline$[8]$ & $\begin{array}{l}\text { Dificuldade na identificação } \\
\text { das funcionalidades }\end{array}$ & {$[4]$} & $\begin{array}{l}\text { A UX pode ser diferente de um } \\
\text { sistema IoT avaliado }\end{array}$ \\
& $\begin{array}{l}\text { individualmente, em relação a } \\
\text { mapeamento delas na interface } \\
\text { do dispositivo móvel. }\end{array}$ & $\begin{array}{l}\text { umbiente com várias } \\
\text { aplicações IoT. }\end{array}$ \\
\hline
\end{tabular}

A partir dos problemas de UX no cenário de IoT identificados na revisão da literatura, iniciamos a elaboração dos itens de 
Towards Developing a Practical Tool to Assist UX Evaluation in the loT Scenario

verificação de um checklist de observação do comportamento do usuário. O checklist de observação foi escolhido por ser uma ferramenta que pode ser empregada com métodos empíricos e auxiliar os avaliadores na condução da avaliação.

Dessa forma, a Tabela 1 exemplifica os tipos de problemas e dificuldades listados na literatura, que deram insumo para a primeira versão do instrumento de avaliação. O foco deste estudo não é ser exaustivo na discussão dos resultados da revisão da literatura, mas na descrição dos aspectos que auxiliaram na construção do instrumento de avaliação de UX no cenário de IoT.

\subsection{Avaliação da UX em um ambiente IoT}

Em paralelo à revisão da literatura, foi realizado uma avaliação de UX com uma aplicação IoT. O principal objetivo desta etapa foi verificar as dificuldades ao realizar a avaliação de UX em um ambiente IoT e quais problemas de UX ocorreriam na aplicação IoT sob avaliação. Isso auxiliou principalmente na visualização das categorias e melhor organização dos aspectos envolvendo UX e IoT. Para alcançar isso, foi utilizado o Automa GREat [1], um aplicativo móvel que realiza o controle de luzes inteligentes (três luzes) e um ar condicionado do ambiente foi avaliado. A Figura 3 apresenta as interfaces do Automa GREat.

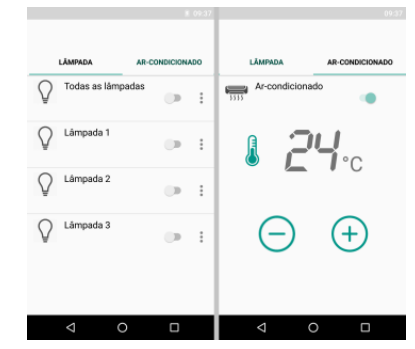

Figura 3: Interfaces do aplicativo Automa GREat.

A avaliação foi organizada em três partes i) assinatura do termo de consentimento e coleta de dados demográficos e experiência com tecnologia por meio de questionário, ii) observação do comportamento do usuário ao realizar tarefas especificas e iii) entrevistas e questionário final.

Após a realização de um teste piloto, a avaliação foi realizada por cinco usuários, sendo quatro homens e uma mulher que executaram um conjunto de seis tarefas em laboratório. Os usuários tinham de 18 a 60 anos, com familiaridade com dispositivos móveis e precisavam saber mexer nas funções básicas de um ar-condicionado.

Os resultados da avaliação são apresentados na Tabela 2. A partir deles, foi possível observar quais aspectos relacionados a atenção do observador deve estar concentrada. Diferente de aplicativos tradicionais, a interface transpõe um único dispositivo e se torna o ambiente como um todo. Tal cenário pode vir à torna a observação da interação mais custosa devido à quantidade de elementos a serem observados. Além disso, foi possível observar como a interação thing-thing pode impactar na UX. Por exemplo, ao apresentar falha na conexão com o arcondicionado, $40 \%$ dos usuários não conseguiram concluir a T5.
WebMedia'2018: Workshops e Pôsteres, Salvador, Brasil

Além disso, o aplicativo não emitia nenhum feedback acerca do problema ao usuário tornando um problema crítico.

Tabela 2: Tarefas realizadas pelos usuários, taxa de completude e exemplos de problemas identificados.

\begin{tabular}{lccc} 
Tarefas & $\begin{array}{c}\text { \% Taxa de } \\
\text { Completude }\end{array}$ & $\begin{array}{l}\text { Exemplo de problema de UX e IoT } \\
\text { identificado }\end{array}$ \\
\hline $\begin{array}{l}\text { T1. Ligar } \\
\text { ar-condicionado. }\end{array}$ & $80 \%$ & $\begin{array}{l}\text { O usuário realizou os passos da tarefa } \\
\text { corretamente. No entanto, a aplicação } \\
\text { falhou em ligar o ar condicionado } \\
\text { causando frustração ao usuário. }\end{array}$ \\
\hline T2. Ligar luzes. & $100 \%$ & $\begin{array}{l}\text { O usuário ficava inseguro quanto ao } \\
\text { feedback de qual das luzes seria acesa no } \\
\text { ambiente ao interagir na interface do } \\
\text { aplicativo. }\end{array}$ \\
\hline $\begin{array}{l}\text { T3. Mudar } \\
\text { a temperatura do } \\
\text { ar-condicionado. }\end{array}$ & $80 \%$ & $\begin{array}{l}\text { O usuário realizou os passos da tarefa } \\
\text { corretamente. No entanto, a aplicação } \\
\text { falhou em ligar o ar condicionado } \\
\text { causando frustração ao usuário. }\end{array}$ \\
\hline $\begin{array}{l}\text { T4. Alterar a } \\
\text { cor das luzes. }\end{array}$ & $80 \%$ & $\begin{array}{l}\text { O usuário não conseguiu identificar como } \\
\text { alterar a cor das lâmpadas. }\end{array}$ \\
\hline $\begin{array}{l}\text { T5. Desligar } \\
\text { ar-condicionado. }\end{array}$ & $60 \%$ & $\begin{array}{l}\text { O usuário realizou os passos da tarefa } \\
\text { corretamente, mas a aplicação não } \\
\text { funcionou ao comando do usuário. }\end{array}$ \\
\hline T6. Desligar luzes. & $100 \%$ & $\begin{array}{l}\text { O usuário teve dificuldade no } \\
\text { mapeamento da interação de qual luz } \\
\text { seria apagada no ambiente ao interagir } \\
\text { com a interface. }\end{array}$ \\
\hline
\end{tabular}

\subsection{Questionário com Especialistas de IoT}

Com base nos resultados da revisão da literatura e da avaliação da UX em um ambiente IoT, um conjunto de 15 itens de verificação para avaliação da UX em ambientes IoT foi proposto. Para refinar e avaliar tais itens, foi enviado um questionário para profissionais e pesquisadores que desenvolvem e avaliam aplicações no contexto IoT. O questionário foi disponibilizado online e era constituído por 14 questões (quatro abertas) organizadas em três seções. A primeira seção abordava questões sobre dados demográficos do especialista; a segunda, sua experiência com IoT; e a terceira, investigava sua percepção sobre um conjunto de itens de verificação gerados com resultados dos métodos apresentados anteriormente neste trabalho. É importante reforçar que aspectos éticos de privacidade e anonimato foram respeitados durante a aplicação do questionário.

Dos 14 respondentes, 64\% apresentava de um a dois anos trabalhando com IoT e $36 \%$ apresentavam experiência de quatro a cinco anos na área. Ainda relacionado à experiência com IoT, os participantes apresentaram uma média de participação do desenvolvimento e/ou avaliação de pelo menos duas aplicações no cenário de IoT, em domínios como smarthomes, mobilidade urbana e saúde. Os especialistas selecionaram os itens de verificação mais importantes ao realizar uma avaliação de UX em um ambiente IoT. Na Tabela 3 são apresentados os pontos do checklist melhor avaliados pelos especialistas. Além da seleção, os especialistas sugeriram melhorias na descrição dos itens e a incorporação de aspectos não contemplados pelos pontos de verificação apresentados no questionário. Dessa forma, os itens 
de verificação passaram a incorporar melhor e de maneira mais clara aspectos relacionados à programabilidade e sincronia de serviços, estratégias de interação, satisfação e prazer no uso.

Tabela 3: Avaliação dos itens de verificação pelos especialistas de IoT.

\begin{tabular}{cl}
\hline \%especialistas & \multicolumn{1}{c}{ Item de verificação (sensibilidade ao contexto / human-thing) } \\
\hline $\mathbf{8 0 \%}$ & $\begin{array}{l}\text { O sistema IoT percebe as mudanças de contexto e se adequa } \\
\text { corretamente as necessidades do usuário. }\end{array}$ \\
\hline $\mathbf{7 0 \%}$ & $\begin{array}{l}\text { O usuário percebeu problemas de funcionamento relacionados a } \\
\text { interação thing-thing (Por exemplo: Problemas de sincronia, } \\
\text { responsividade e conectividade das “coisas"). }\end{array}$ \\
\hline $\mathbf{6 0 \%}$ & $\begin{array}{l}\text { O usuário demonstra sinais de desconforto ou incômodo com o } \\
\text { sistema IoT (Sinais de cansaço, insegurança durante a interação). }\end{array}$ \\
\hline $\mathbf{6 0 \%}$ & $\begin{array}{l}\text { O usuário demonstra sinais de frustração durante a interação (Por } \\
\text { exemplo: expressões de descontentamento ou tristeza). }\end{array}$ \\
\hline
\end{tabular}

\subsection{Discussão}

Todas as situações apresentadas anteriormente pela aplicação de cada método auxiliaram na construção e definição do instrumento de avaliação. A revisão da literatura e avaliação de UX auxiliaram no levantamento dos primeiros pontos de verificação relacionados principalmente as interações (thingthing e human-thing) e as características de IoT que vem ganhando destaque como, sensibilidade ao contexto e programabilidade. Além disso, a definição por um checklist seguiu a necessidade de observar o comportamento do usuário como principal meio de obter evidências sobre a UX em um cenário de IoT. Tais percepções capturadas pela literatura foram também identificadas na avaliação do Automa GREat.

$\mathrm{O}$ questionário com especialistas em IoT auxiliou na organização, na priorização e melhoria dos itens do checklist. É importante observar que o conjunto final itens de verificação são mais voltados ao contexto de casas e prédios inteligentes. Isso ocorreu devido a forte influência dos estudos da revisão da literatura, da avaliação de UX e do conhecimento maior dos especialistas em soluções nesse domínio. Tal cenário não limita a avaliações de soluções nesse domínio, mas evidência a fase em desenvolvimento em que se encontra a ferramenta. Dessa forma, é necessário que o instrumento seja aplicados em avaliações de UX no cenário IoT para que possa se obter as melhorias necessárias ao checklist.

\section{CHECKLIST PARA AVALIAÇÃO DE UX NO CENÁRIO DE IOT}

A ferramenta apresenta 19 itens de verificação organizados em duas categorias de interação (human-thing e thing-thing) presentes em um ambiente IoT, como discutido por Andrade et al. (2017) [1] e Roland et al. (2015) [11]. Essa organização foi escolhida por auxiliar os avaliadores na imersão e na compreensão do ambiente IoT pela perspectiva do usuário. Além disso, os problemas e melhorias identificados por cada categoria podem facilitar a evolução do sistema IoT por parte dos desenvolvedores.

A categoria thing-thing se concentra na verificação de problemas entre as "coisas" entre si e a Internet. Seus seis itens estão organizados nas subcaracterísticas de sensibilidade ao contexto e programabilidade. Os itens descritos na categoria human-thing seguem uma organização similar, porém com a diferença de uma subcategoria a mais, aspectos gerais da UX. Os treze pontos de verificação apresentados na categoria humanthing são orientados em observar o nível de satisfação da experiência do usuário ao interagir no ambiente IoT. As categorias e subcategorias foram aspectos que ganharam destaque durante todo o processo de construção do checklist como descrito anteriormente na Seção 3.

Um fragmento do checklist é apresentado na Tabela 4 com categorias, subcategorias e quatro itens de verificação. $\mathrm{O}$ checklist completo pode ser acessado em: https://goo.gl/xpq7w3. $\mathrm{Na}$ Tabela 4 os itens quatro e cinco estão relacionados a identificação da utilidade, controle e compreensão do usuário sobre o ambiente IoT. Tais aspectos se demonstram primordiais para observação inicial, por conseguinte para a identificação da qualidade da UX em ambiente IoT. Os outros itens (14 e 19) se relacionam já ao atendimento das necessidades reais do usuário com o uso da aplicação. É importante ressaltar que o checklist está em processo de construção e o próximo passo no seu desenvolvimento é a aplicação em avaliações de UX no cenário IoT.

\section{Tabela 4: Fragmento do Checklist de Observação da UX em cenário IoT}

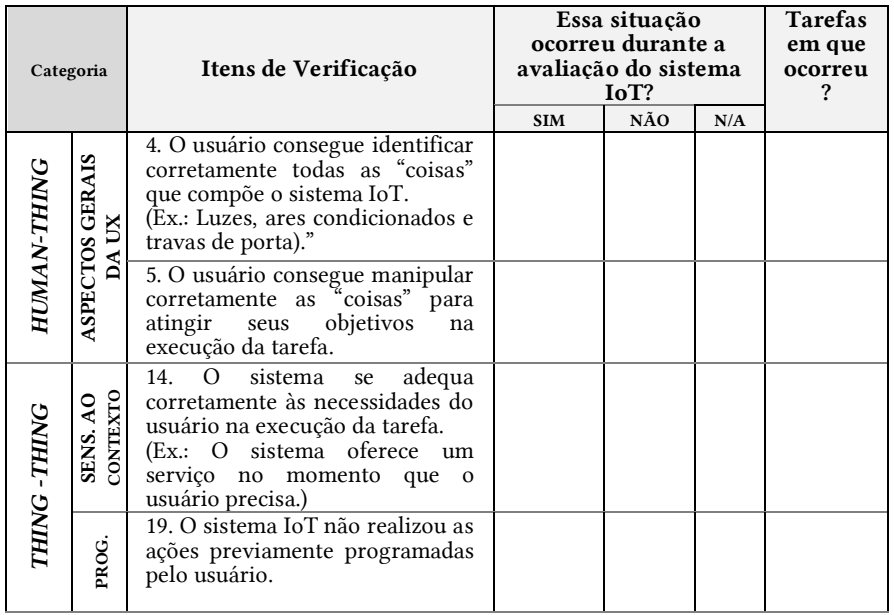

\subsection{Descrição de Uso da Ferramenta}

O checklist precisa ser aplicado no mínimo por dois avaliadores, um responsável pela condução das tarefas a serem realizadas pelos usuários e outro avaliador dedicado a observação e preenchimento da ferramenta. Os avaliadores devem previamente verificar se a aplicação IoT apresenta as características de sensibilidade ao contexto e programabilidade e se todos os itens de verificação presentes do checklist serão observados na avaliação. Caso se identifique que algum item não se aplica ao escopo da avaliação, o observador deve marcar 
Towards Developing a Practical Tool to Assist UX Evaluation in the loT Scenario

previamente na ferramenta a opção "não se aplica (N/A)" para evitar distrações durante a observação. É recomendado que o observador estude a ferramenta e obtenha familiaridade com os itens de verificação. A realização da avaliação piloto pode auxiliar na familiaridade dos avaliadores com os pontos de verificação do checklist.

Recomenda-se o início do preenchimento da ferramenta pelos itens da categoria human-thing que estão relacionado ao contato direto do usuário com as "coisas" a fim de cumprir o conjunto de tarefas estabelecidas na avaliação. A subcategoria "aspectos gerais da UX" pode ser avaliada para todo o conjunto de tarefas do experimento. Já as subcategorias de "sensibilidade ao contexto" e "programabilidade" podem ser observadas em tarefas que propiciem sua observação em ambas as categorias. Dessa forma, recomenda-se que os avaliadores preparem o ambiente da avaliação para que consiga reproduzir situações contextuais chaves para o funcionamento da aplicação IoT, sejam elas realizadas em laboratório ou no contexto real de uso.

Após o preenchimento dos itens da primeira categoria, devem ser preenchidos os itens da thing-thing. Se sugere indicar no planejamento da avaliação a marcação das tarefas diretamente relacionadas a verificação desses itens. A reprodução das situações contextuais será primordial para a observação desses itens. É importante reforçar que os itens dessa categoria, se relacionam ao funcionamento do ambiente IoT e não são diretamente relacionadas as ações do usuário.

A interpretação do checklist pode ser realizada pela identificação dos itens mais recorrentes e críticos a UX. Após a delineação da porção mais crítica, deve-se sugerir um conjunto de recomendações que auxiliem nas melhorias do sistema IoT, tendo em vista a melhoria da UX. Caso mais de um observador realize o preenchimento da ferramenta, os mesmos devem se reunir e consolidar os resultados da ferramenta.

Recomenda-se o uso da técnica Thinking aloud [7] para auxiliar na compreensão das atitudes e ações do usuário. A técnica precisa ser estimulada pelo condutor das tarefas do usuário e registrada pelo observador que deve realizar o preenchimento da ferramenta. Assim como algum sistema de log que auxilie na identificação dos problemas na thing-thing, visto que pode ser difícil ao avaliador perceber todos utilizando só observação.

\section{CONCLUSÃO}

Este trabalho apresentou a construção de um checklist para auxiliar avaliadores iniciantes a realizarem avaliação de UX no cenário de IoT. O checklist foi criado baseado na metodologia de Stufflebeam (2000) [13], onde foram criados os itens de verificação a partir dos resultados da revisão da literatura e de uma avaliação de UX no cenário de IoT. O artefato foi revisado e validado por 14 especialistas em IoT por meio de um questionário online. A versão atual da ferramenta apresenta um conjunto de 19 itens organizados nos tipos de interação presentes em um ambiente IoT. Como trabalho futuro pretendese aplicar a ferramenta em avaliações de UX no cenário IoT. Com
WebMedia'2018: Workshops e Pôsteres, Salvador, Brasil

isso, refinar o checklist, obtendo amadurecimento e evolução da ferramenta.

\section{AGRADECIMENTOS}

Rodrigo L. A. Almeida, Ticianne G. R. Darin, Italo L. de Araújo agradecem a FCPC pelas bolsas financiadas através de recursos da Lei de Informática e ao CNPq por patrocinar Rossana Maria de Castro Andrade com uma bolsa de produtividade DT-2, número de processo 314021/2009-4. Italo L. de Araújo agradece a CAPES pelo apoio recebido na sua pesquisa de doutorado.

\section{REFERÊNCIAS}

1. Rossana M.C. Andrade, Rainara M. Carvalho, Italo Linhares de Araújo, Káthia M. Oliveira, and Marcio E.F. Maia. 2017. What changes from ubiquitous computing to internet of things in interaction evaluation? In Lecture Notes in Computer Science (including subseries Lecture Notes in Artificial Intelligence and Lecture Notes in Bioinformatics), 3-21. https://doi.org/10.1007/978-3-319-586977_1

2. Luigi Atzori, Antonio Iera, and Giacomo Morabito. 2010. The internet of things: A survey. Computer networks 54, 15: 2787-2805.

3. Johanna Bergman and Isabelle Johansson. 2017. The User Experience Perspective of Internet of Things Development.

4. Hao Cai, Egon Toft, Ole Hejlesen, John Hansen, Claus Oestergaard, and Birthe Dinesen. 2015. Health professionals' user experience of the intelligent bed in patients' homes. International Journal of Technology Assessment in Health Care 31, 4: 256-263. https://doi.org/10.1017/S0266462315000380

5. Elizabeth Sucupira Furtado, Gilberto Villa Nova, Isabela Gasparini, Leonardo Cunha de Miranda, Marcelo Barbosa, Tatiana Silva de Alencar, and Vânia Paula de Almeida Neris. 2017. Re-evaluating the First Five Years of the GrandIHC-Br-Challenge 3: Ubiquity, Multiple Devices and Tangibility. In Proceedings of the XVI Brazilian Symposium on Human Factors in Computing Systems, 69.

6. Jayavardhana Gubbi, Rajkumar Buyya, Slaven Marusic, and Marimuthu Palaniswami. 2013. Internet of Things ( IoT ): A vision, architectural elements, and future directions. Future Generation Computer Systems 29, 7: 1645-1660 https://doi.org/10.1016/j.future.2013.01.010

7. D Hauser. 1989. Thinking aloud. Heart Lung 18, 3: 321-322. Retrieved from http://www.ncbi.nlm.nih.gov/entrez/query.fcgi?cmd=Retrieve\&db=PubMed\&d opt=Citation\&list_uids $=2722544$

8. Lasse Kaila, Henrik Raula, Miika Valtonen, and Karri Palovuori. 2012. Living wood: a self-hiding calm user interface. In Proceeding of the 16th International Academic MindTrek Conference, 267-274.

9. Ibrahim Mashal, Osama Alsaryrah, Tein Yaw Chung, Cheng Zen Yang, Wen Hsing Kuo, and Dharma P. Agrawal. 2015. Choices for interaction with things on Internet and underlying issues. Ad Hoc Networks 28: 68-90. https://doi.org/10.1016/j.adhoc.2014.12.006

10. Alessandra Papetti, Andrea Capitanelli, Lorenzo Cavalieri, Silvia Ceccacci, Francesca Gullà, and Michele Germani. 2016. Consumers vs Internet of Things: A Systematic Evaluation Process to Drive Users in the Smart World. In Procedia CIRP, 541-546. https://doi.org/10.1016/j.procir.2016.04.128

11. Claire Rowland, Elizabeth Goodman, Martin Charlier, Alfred Lui, and Ann Light. 2015. Designing Connected Products: UX for the Consumer Internet of Things. " O'Reilly Media, Inc."

12. Vagner F de Santana, Vânia Neris, Kamila R H Rodrigues, Renata Oliveira, and Newton Galindo Jr. 2017. Activity of Brazilian HCI Community from 2012 to 2017 in the Context of the Challenge'Future, Smart Cities, and Sustainability'. In Proceedings of the XVI Brazilian Symposium on Human Factors in Computing Systems, 67.

13. Daniel L Stufflebeam. 2000. Guidelines for developing evaluation checklists: the checklists development checklist (CDC). Kalamazoo, MI: The Evaluation Center. Retrieved on fanuary 16, July: 2008. Retrieved from http://www.wmich.edu/evalctr/archive_checklists/guidelines_cdc.pdf $\% 5$ Cnpap ers3://publication/uuid/293B1EC6-6CA7-4CD1-9E80-885BC85B06A4

14. Jean-Philippe Vasseur and Adam Dunkels. 2010. Interconnecting Smart Objects with IP: The Next Internet (Google eBook). Morgan Kaufmann. Retrieved from http://books.google.com/books?hl=en\&lr=\&id=mVji-YA5kgEC\&pgis=1

15. Ovidiu Vermesan, Peter Friess, Patrick Guillemin, Sergio Gusmeroli, Sundmaeker Harald, Alessandro Bassi, Ignacio Soler Jubert, Margaretha Mazura, Mark Harrison, Markus Eisenhauer, and Pat Doody. 2011. Internet of Things Strategic Research Roadmap. IoT European Research Cluster, 9-52. 